\title{
ANTIMONOTONICITY: CONCURRENT CREATION AND ANNIHILATION OF PERIODIC ORBITS
}

\author{
I. KAN AND J. A. YORKE
}

\begin{abstract}
One-parameter families $f_{\lambda}$ of diffeomorphisms of the Euclidean plane are known to have a complicated bifurcation pattern as $\lambda$ varies near certain values, namely where homoclinic tangencies are created. We argue that the bifurcation pattern is much more irregular than previously reported. Our results contrast with the monotonicity result for the wellunderstood one-dimensional family $g_{\lambda}(x)=\lambda x(1-x)$, where it is known that periodic orbits are created and never annihilated as $\lambda$ increases. We show that this monotonicity in the creation of periodic orbits never occurs for any one-parameter family of $C^{3}$ area contracting diffeomorphisms of the Euclidean plane, excluding certain technical degenerate cases where our analysis breaks down. It has been shown that in each neighborhood of a parameter value at which a homoclinic tangency occurs, there are either infinitely many parameter values at which periodic orbits are created or infinitely many at which periodic orbits are annihilated. We show that there are both infinitely many values at which periodic orbits are created and infinitely many at which periodic orbits are annihilated. We call this phenomenon antimonotonicity.
\end{abstract}

\section{INTRODUCTION}

The orbit of point $x$ under a diffeomorphism of the plane $f$ is the sequence $\left\{f^{k}(x)\right\}$, where for $k \geq 0, f^{k}$ denotes the $k$-fold composition of $f, f^{-k}$ denotes the $k$-fold composition of $f^{-1}$ and $f^{0}$ is the identity map. Let $p$ be a periodic point with period $n$. The stable manifold $W^{s}(p)$ of the point $p$ is the set $\{x$ : $\left.\lim _{k \rightarrow \infty} f^{n k}(x)=p\right\}$. Similarly, the unstable manifold $W^{u}(p)$ of $p$ is $\left\{x: \lim _{k \rightarrow \infty} f^{-n k}=p\right\}$. We assume that $p$ is a hyperbolic saddle, that is, the eigenvalues $e_{1}, e_{2}$ of $D f^{n}(p)$ are such that $\left|e_{1}\right|<1<\left|e_{2}\right|$. Since $f$ is a diffeomorphism of the plane, both $W^{s}(p)$ and $W^{u}(p)$ are curves. There exists a homoclinic tangency

Received by the editors November 2, 1988 and, in revised form, May 1, 1989. 1980 Mathematics Subject Classification (1985Revision). Primary 54C35, 58 F13. Partial support provided by DARPA/ACMP program and by AFOSR-81-0217. 
of $p$ at $q$ if $W^{s}(p)$ and $W^{u}(p)$ intersect tangentially at $q$. The homoclinic tangency of $p$ at $q$ for a one-parameter family $f_{\lambda}$ at $\lambda=\lambda_{0}$ is called nondegenerate if $W^{s}(p)$ and $W^{u}(p)$ have quadratic contact at $q$ and $W^{s}(p)$ has nonzero velocity transverse to $W^{u}(p)$ at $q$ as $\lambda$ varies [R]. Any value $\lambda_{0}$ at which this occurs is called a nondegenerate tangency value.

A one-parameter family of maps $g_{\lambda}$ is called monotone increasing (decreasing) on an interval $J$ of parameter values if there are no bifurcations for $\lambda \in J$ in which periodic orbits are annihilated as $\lambda$ increases (decreases, respectively). We say $f_{\lambda}$ is antimonotone at $\lambda_{0}$ if periodic orbits are both created and annihilated as $\lambda$ increases in each neighborhood of the parameter value $\lambda_{0}$.

The only smooth family for which monotonicity has been proved is the quadratic family $g_{\lambda}(x)=\lambda x(1-x)$ (Douady, Hubbard, Milnor, Thurston, Sullivan, see [MT]). By contrast we have the following theorem.

Antimonotonicity Theorem. Each dissipative $C^{3}$ planar diffeomorphism family is antimonotone at each nondegenerate homoclinic tangency value.

Note that this result says nothing about what happens near degenerate homoclinic tangency values, but we believe this situation is essentially the same as for the nondegenerate case.

We sketch the proof for a model case. A paper detailing the proof of the general result is in preparation. If two curves are tangent at $\lambda=\lambda_{0}$ and move apart, so that they do not intersect as $\lambda$ increases (decreases) beyond $\lambda_{0}$, then we say contact is broken at $\lambda_{0}$ (contact is made at $\lambda_{0}$, respectively), and we say $\lambda_{0}$ is a contact-breaking value (contact-making value, respectively).

Bubble Lemma. If $\lambda_{0}$ is a nondegenerate tangency value at which contact is made, then there are nondegenerate tangency values arbitrarily close to $\lambda_{0}$ at which contact is broken (and vice versa).

The theorem follows immediately from the Bubble Lemma because in each neighborhood of a contact-making nondegenerate tangency value, infinitely many periodic orbits are created (and near contact-breaking ones, infinitely many are annihilated) [N, GS]. Thus, in each neighborhood of a nondegenerate tangency, orbits are both created and annihilated, as is illustrated in Figure 1 for the example of the Henon family. 


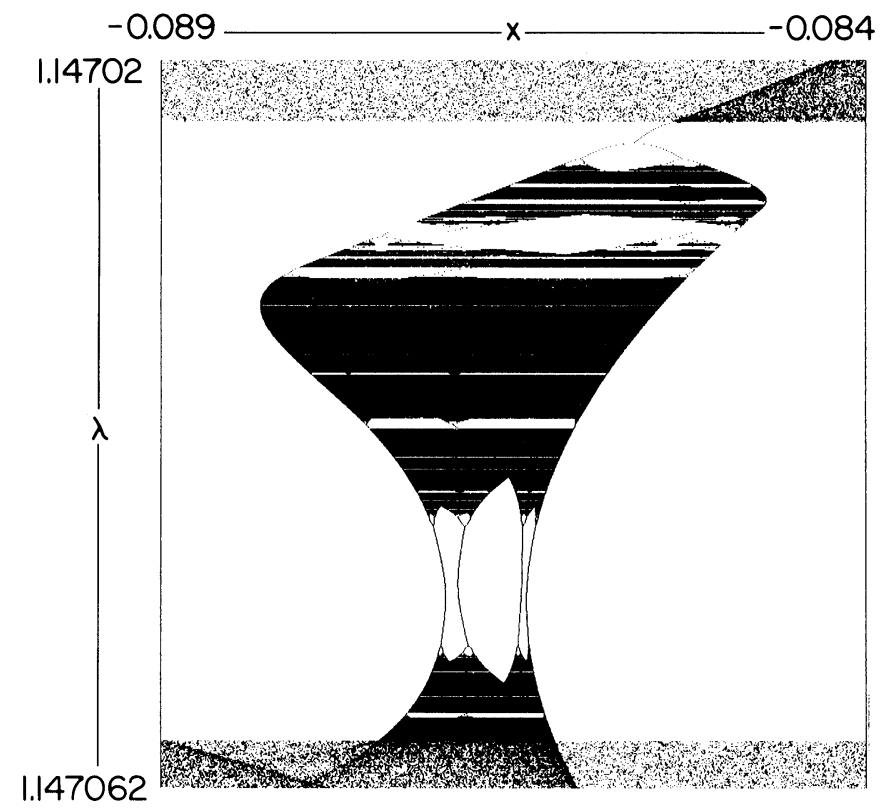

Figure 1. Small bubble in Henon family $H_{\lambda}(x, y)=$ $\left(\lambda-x^{2}+0.3 y, x\right) .5,000$ PREITERATES, $x$-COORDINATE OF 80,000 ITERATES PLOTTED PER $\lambda$ VALUE.

\section{Preliminaries}

For each Cantor set $C \subset \mathbb{R}$ Newhouse [N] defines a number in $[0, \infty)$ called the thickness $\tau(C)$ associated with $C$. A "middle$\theta$ " Cantor set $C_{\theta}=I \backslash G_{\alpha}$ is constructed inductively as follows: $I=[0,1]$ and $I_{\alpha, 0}$ and $I_{\alpha, 1}$ are the left and right component of $I_{\alpha} \backslash G_{\alpha}$, respectively, where $G_{\alpha}$ is an open interval of length $\theta \cdot\left|I_{\alpha}\right|$ in the middle of $I_{\alpha}$. The thickness of $C_{\theta}$ is $(1-\theta) / 2 \theta$. Newhouse proves the following lemma.

Thickness Lemma. Let $F$ and $H$ be Cantor sets in $R$, with $H \cap$ $\operatorname{hull}(F)$ and $\operatorname{hull}(H) \cap F$ both nonempty, and $\tau(H) \cdot \tau(F)>1$. Then $H \cap F$ is nonempty.

A Newhouse horseshoe family $N_{\lambda}$ is defined as follows. (See Figure 2 on page 472 for symbols, coordinates, and the role of the constants, and see Figure 3 on page 472 for the first iterate 


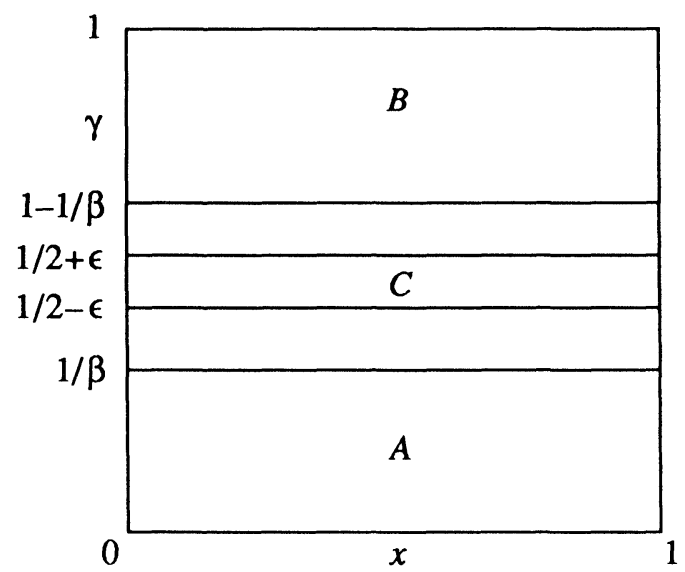

FIGURE 2. COORDINATES FOR $N_{\lambda}$.

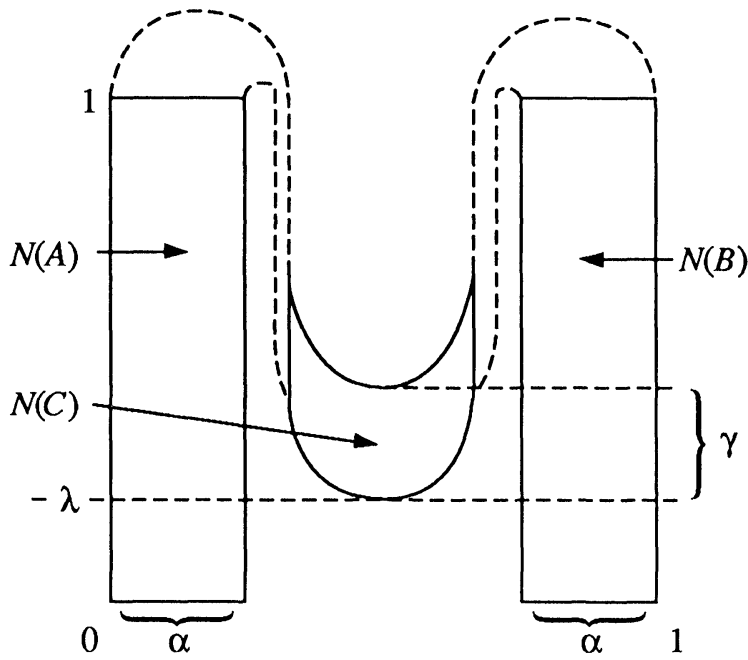

Figure 3. First ITERATE OF $N_{\lambda}$.

$\left.N_{\lambda}\right)$. Define $N_{\lambda}(x, y)=(\alpha x, \beta y)$ for $(x, y) \in A ; N_{\lambda}(x, y)=$ $(1-\alpha x, \beta(1-y))$ for $(x, y) \in B ; N_{\lambda}(x, y)=(y,-\lambda+\gamma(1-x)$ $\left.+\delta(y-1 / 2)^{2}\right)$ for $(x, y) \in C$; and continue $N_{\lambda}$ smoothly to the rest of $\mathbb{R}^{2}$.

We choose $\alpha \beta<1$ so $N_{\lambda}$ is dissipative (i.e. $\left|\operatorname{det} D\left(N_{\lambda}\right)\right|<1$ ) throughout $A \cup B$, and we choose $\alpha, \beta, \gamma, \delta, \varepsilon$ such that $N_{\lambda}$ is one-to-one on $A \cup B \cup C$. This implies $\beta>2$. Let $\Lambda$ denote the maximal invariant subset of $A \cup B ; \Lambda$ is a Cantor set and is the product $\Lambda_{u} \times \Lambda_{s}$ of two Cantor sets. $\Lambda_{u}$ is the projection of $\Lambda$ 
onto the $x$-axis and $\Lambda_{s}$ onto the $y$-axis. We assume that $\alpha$ and $\beta$ are selected so that $\tau\left(\Lambda_{s}\right) \cdot \tau\left(\Lambda_{u}\right)=(\beta-2)^{-1} \alpha(1-2 \alpha)^{-1}>1$.

A primary stable (unstable) segment is a line segment of the form $[0,1] \times\{y\}$ where $y \in \Lambda_{s},\left(\{x\} \times[0,1]\right.$ where $x \in \Lambda_{u}$, respectively). A primary unstable parabola is a parabolic arc of the form $N_{\lambda}(x,[1 / 2-\varepsilon, 1 / 2+\varepsilon])$ where $x \in \Lambda_{u}$.

Newhouse and Robinson show in [N, R], that in effect, there exist parameter values $\lambda$ near homoclinic tangencies where for a proper choice of coordinates the map is similar to Figure 3. We are assuming that the map changes in a regular way as $\lambda$ varies, thereby avoiding technical complications.

\section{Proof of Bubble Lemma ASSUMING NEWHOUSE HORSESHOE FAMILIES OCCUR}

Let $\lambda_{0}$ be a nondegenerate tangency value, which we assume to be a contact-making tangency. We assume that on a small interval, arbitrarily near $\lambda_{0}$, there is a Newhouse horseshoe family. We rescale that small interval to be $[0,1]$. The primary tangencies (the tangencies of primary parabolas with primary stable segments) are all contact-making. We will show that arbitrarily near $\lambda=0$, there is a nondegenerate tangency which is contact-breaking and is not primary.

The parabolic arc of the form $v(t, \xi)=\left(1 / 2+t, \beta^{-n}+\xi+\delta t^{2}\right)$ for $0<\xi<(1-2 / \beta) \beta^{1-n}, \delta t^{2}<\beta^{-n}-2 \beta^{-n-1}-\xi,|t|<\varepsilon$, lies in a gap in the Cantor set of primary stable leaves as shown in Figure 4.

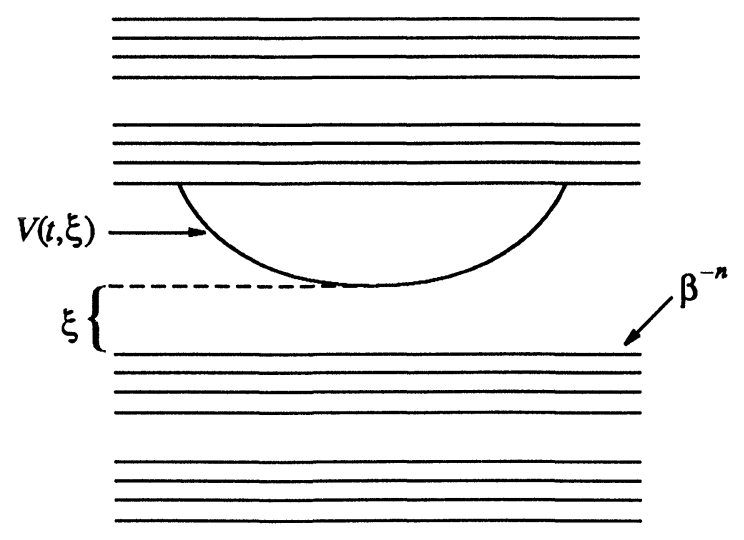

Figure 4. THE ARC $v(t, \xi)$. 
Let $\Gamma(\xi)$ denote the set of parameters such that $v(t, \xi)$ lies on a primary parabola. For each $\lambda$ the vertices of the primary parabolas have $y$-coordinates at $\left(-\lambda+\gamma \Lambda_{u}\right)$, so we see that $\Gamma(\xi)=$ $-\gamma \Lambda_{u}-\xi-\beta^{-n}$ and the thickness of $\Gamma(\xi)$ is equal to $\tau\left(\Lambda_{u}\right)$. The $n$th image of $v(t, \xi)$ under $N_{\lambda}$ is

$$
\begin{aligned}
v_{n}(t, \xi, \lambda)= & \left(\beta^{-1}+\xi \beta^{n-1}+\delta \beta^{n-1} t^{2},\right. \\
& -\lambda-\gamma \alpha^{n-1}(1 / 2+t)+\gamma \\
& \left.+\delta\left(\beta^{-1}+\xi \beta^{n-1}+\delta^{n-1} t^{2}-1 / 2\right)^{2}\right) .
\end{aligned}
$$

There is a $\xi=\bar{\xi}, t=\bar{t}$ at which the $y$-coordinate has a stationary inflection point as shown in Figure $5 \mathrm{~b}$, and $\bar{\xi}$ and $\bar{t}$ satisfy $4 \delta \beta^{n-1}\left(\beta^{-1}+\bar{\xi} \beta^{n-1}-1 / 2\right)=-3\left(\gamma \alpha^{n-1} \beta^{n-1}\right)^{2 / 3}$, and $\bar{t}=$ $\left(-\gamma \alpha^{n-1} \beta^{n-1}\right)^{2 / 3} / 4 \delta \beta^{n-1}$. Notice

$$
\bar{\xi}=(1 / 2)(1-2 / \beta) \beta^{1-n}+(\alpha \beta)^{2 n / 3} 0\left(\beta^{-2 n}\right)
$$

so for large $n$ we have $0<\bar{\xi}<(1-2 / \beta) \beta^{1-n}$.

Claim. For fixed $\xi \leq \bar{\xi}$, with $\bar{\xi}-\xi$ sufficiently small, there exists a $\hat{\lambda} \in \Gamma(\xi)$ such that the $n$th iterate of the primary parabola containing $v(t, \xi)$ has a tangency with a primary stable segment. This tangency is contact-breaking and is nondegenerate for $\xi<\bar{\xi}$.

The first part of this claim follows from the fact that the local maximum $y(\xi, \lambda)$ (see Figure $5 \mathrm{a}$ ) of the $y$-coordinate of $v_{n}(t, \xi, \lambda)$ depends linearly on $\lambda$. That is,

$$
\{y(\xi, \lambda) \mid \lambda \in \Gamma(\xi)\}=\{y(\xi, 0)-\lambda \mid \lambda \in \Gamma(\xi)\},
$$

and so $\{y(\xi, \lambda) \mid \lambda \in \Gamma(\xi)\}$ has thickness $\tau\left(\Lambda_{u}\right)$. By the Thickness Lemma, there exists some $\hat{\lambda} \in \Gamma(\xi)$ such that $y(\xi, \hat{\lambda}) \in \Lambda_{s}$. Note that $\hat{\lambda}$ is $0\left(\beta^{-n}\right)$. Since $\hat{\lambda}$ is in $\Gamma(\xi)$, there is a primary unstable parabola which contains $v(t, \xi)$, so $v_{n}(t, \xi, \hat{\lambda})$ is contained in the unstable manifold of $\Lambda$ and is tangent to a primary stable segment of $\Lambda$. As $\lambda$ varies near 0 , the position of this primary unstable parabola is $v(t, \xi+\lambda)$. Nondegeneracy and contact-breaking can be verified by considering the $y$-coordinate of $d\left(v_{n}(t, \xi+\lambda, \lambda)\right) / d \lambda$ and noting that for sufficiently small $\bar{\xi}-\xi>0$ and large $n$ this derivative is negative for $t$ sufficiently close to $\bar{t}$.

We have shown that there is a primary stable leaf $S$ and a primary unstable parabola $U$ so that the $n$th iterate of $U$ has a 


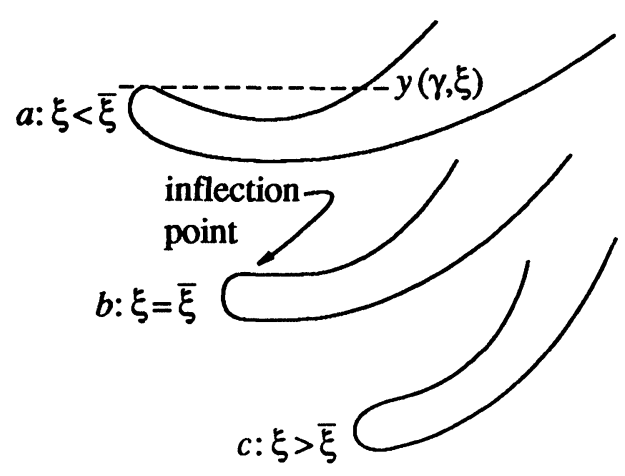

Figure 5. The inflection VAlue $\bar{\xi}$.

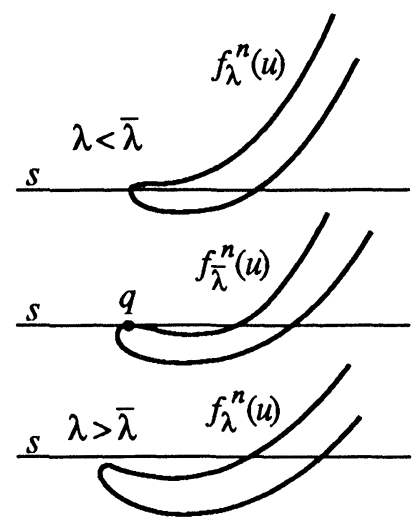

Figure 6. Contact-Breaking tangenCy $q$ AT $\lambda=\hat{\lambda}$.

contact-breaking tangency with $S$ (see Figure 6). Since the stable and unstable manifold of the fixed point $p$ at $(0,0)$ contain curves arbitrarily close to $S$ and $U$, respectively, we see that $p$ will have contact-breaking tangencies at parameter values arbitrarily near $\hat{\lambda}$. Finally, for $n$ large, this $\hat{\lambda}$ is near 0 .

\section{REFERENCES}

[GS] N. K. Gavrilov and L. P. Šilnikov, On three-dimensional dynamical systems close to systems with structurally unstable homoclinic curve, I, II, Math. USSR-Sb. 88(4) (1972), 467-485, ibid. 90(1) (1973), 139-156.

[MT] J. Milnor and W. P. Thurston, On iterated maps of the interval, Dynamical Systems: Proc. Univ. Maryland 1986-87, Lecture Notes in Math., vol. 1342, Springer-Verlag, Berlin and New York, 1989, pp. 465-563. 
[N] S. Newhouse, The abundance of wild hyperbolic sets and nonsmooth stable sets for diffeomorphisms, Inst. Hautes Etudes Sci. Publ. Math. 50 (1978), 101-151.

[R] C. Robinson, Bifurcation to infinitely many sinks, Comm. Math. Phys. 90 (1983), 433-459.

Department of Mathematics, George Mason University, Fairfax, Virginia 22030 and Naval Surface Warfare Center, White Oak, Maryland

Institute for Physical Science and Technology, University of Maryland, College Park, Maryland 20742 